Linköping Studies in Science and Technology.

Dissertations, No. 1921

\title{
Uncertainty quantification for wave propagation and flow problems with random data
}

\author{
Markus Wahlsten
}

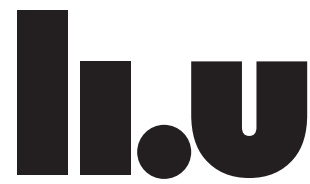

LINKÖPING UNIVERSITY

Department of Mathematics, Division of Computational Mathematics Linköping University, SE-581 83 Linköping, Sweden

Linköping 2018 
This work was funded by the European Union's Seventh Framework Programme for research, technological development and demonstration under grant agreement NO. ACP3-GA-2013-605036.

Linköping Studies in Science and Technology. Dissertations, No. 1921

Uncertainty quantification for wave propagation and flow problems with random data

Copyright (C) Markus Wahlsten, 2018

Division of Computational Mathematics

Department of Mathematics

Linköping University

SE-581 83, Linköping, Sweden

markus. wahlsten@liu.se

ww.liu.se/mai/ms

Typeset by the author in $\mathrm{HT}_{\mathrm{E}} \mathrm{X} 2 \mathrm{e}$ documentation system.

ISSN 0345-7524

ISBN 978-91-7685-339-9

Printed by LiU-Tryck, Linköping, Sweden 2018 
To my family. 

The good thing about science is that it's true whether or not you believe in it.

\section{NEIL DEGRASSE TYSON}





\section{Abstract}

In this thesis we study partial differential equations with random inputs. The effects that different boundary conditions with random data and uncertain geometries have on the solution are analyzed. Further, comparisons and couplings between different uncertainty quantification methods are performed. The numerical simulations are based on provably strongly stable finite difference formulations based on summation-by-parts operators and a weak implementation of boundary and interface conditions.

The first part of this thesis treats the construction of variance reducing boundary conditions. It is shown how the variance of the solution can be manipulated by the choice of boundary conditions, and a close relation between the variance of the solution and the energy estimate is established. The technique is studied on both a purely hyperbolic system as well as an incompletely parabolic system of equations. The applications considered are the Euler, Maxwell's, and NavierStokes equations.

The second part focuses on the effect of uncertain geometry on the solution. We consider a two-dimensional advection-diffusion equation with a stochastically varying boundary. We transform the problem to a fixed domain where comparisons can be made. Numerical results are performed on a problem in heat transfer, where the frequency and amplitude of the prescribed uncertainty are varied.

The final part of the thesis is devoted to the comparison and coupling of different uncertainty quantification methods. An efficiency analysis is performed using the intrusive polynomial chaos expansion with stochastic Galerkin projection, and non-intrusive numerical integration. The techniques are compared using the non-linear viscous Burgers' equation. A provably stable coupling procedure for the two methods is also constructed. The general coupling procedure is exemplified using a hyperbolic system of equations. 



\section{Sammanfattning på svenska}

Många modeller som beskriver fysikaliska, kemiska och biologiska fenomen baseras på lösning av partiella differentialekvationer. Typiska tillämpningsområden är strömningsmekanik, elektromagnetism, ljudutbredning och värmeledning. Gemensamt för de flesta tillämpningarna är att parametrarna och/eller indata till modellerna innehåller osäkerheter i någon form.

Fältet som beskriver osäkerheter i utdata från en modell, givet osäkerheter i dess indata kallas osäkerhetskvantifiering (eng. "uncertainty quantification"). De metoder som används inom osäkerhetskvantifiering kan i huvudsak delas in i två kategorier: intrusiva (eng. "intrusive") och icke-intrusiva (eng. "nonintrusive"). Icke-intrusiva metoder löser originalproblemet flertalet gånger med olika indata, vilket betyder att det ursprungliga deterministiska datorprogrammet kan användas. Intrusiva metoder kräver i sin tur en omskrivning av det ursprungliga problemet, vilket betyder att ett nytt program behöver utvecklas.

Den första delen av avhandlingen behandlar variansreduktion. Eftersom olika randvillkor ger upphov till olika spridning av osäkerheterna på lösningen konstrueras variansreducerande randvillkor, dvs randvillkor med minimal varians. Undersökningen av dessa randvillkor är gjord för Eulers, Maxwells och NavierStokes ekvationer som bland annat används inom strömningsmekanik och elektromagnetism.

Avhandlingens andra del består av en undersökning av effekterna på lösningen vid användning av osäkra geometrier. Osäkerheter i geometrier uppkommer från t.ex. inkorrekta nätgeneratorer eller felaktiga tillverkningsmaskiner. I avhandlingen utvecklas en metod för att analysera och kvantifiera de effekter som uppstår. Effekterna av osäkerheterna studeras och jämförs för ett problem inom värmeöverföring.

Den sista delen i avhandlingen behandlar effektiviteten samt kopplingen mellan intrusiva och icke-intrusiva osäkerhetskvantifieringsmetoder. En prestandautvärdering av de båda metoderna för olika typer av problem presenteras. En teknik där de båda metoderna kopplas i rummet introduceras. Kopplingen medför att beräkningsdomänen kan delas upp i flera delar och de olika metoderna kan användas där de är mest effektiva. Ett exempel på tillämpning är när man simulerar luftflödet kring en flygplansvinge, där luftflödet beter sig annorlunda nära vingen jämfört med långt ut i atmosfären. 



\section{List of Papers}

This thesis is based on the following papers, which from now on will be referred to by their roman numerals:

I. Nordström, J. \& Wahlsten, M. Variance reduction through robust design of boundary conditions for stochastic hyperbolic systems of equations. Journal of Computational Physics, 282, 1-22, (2015).

II. Wahlsten, M. \& Nordström, J. Robust Boundary Conditions for Stochastic Incompletely Parabolic Systems of Equations. Linköping University Press, LiTH-MAT-R--2016/19--SE (Submitted).

III. Wahlsten, M. \& Nordström, J. The effect of uncertain geometries on advection-diffusion of scalar quantities. BIT Numerical Mathematics, 121, (2017).

IV. Wahlsten, M. \& Nordström, J. Stochastic Galerkin Projection and Numerical Integration for Stochastic Investigations of the Viscous Burgers' Equation. Linköping University Press, LiTH-MAT-R--2018/02--SE (Submitted).

V. Wahlsten, M., Ålund, O. \& Nordström, J. An efficient hybrid method for uncertainty quantification. Linköping University Press, LiTH-MAT-R-2018/03--SE (Submitted).

The theoretical analysis has been developed in close collaboration with the coauthor Prof. Jan Nordström and for Paper V, Oskar Ålund. I have written the manuscript with editorial help from the corresponding co-authors Prof. Jan Nordström and for Paper $V$, Oskar Ålund. I have conducted all numerical experiments. 



\section{Acknowledgements}

First of all, I would like express my deepest gratitude to my supervisor Prof. Jan Nordström for his excellent guidance during my PhD. His unbelievable level of commitment, determination and patience has been far beyond my expectations. I've really appreciated receiving constant feedback of my work as well as the interesting projects I've been assigned. It goes without saying that his supervision has been the key to any success I've enjoyed during this period. I should also thank my co-supervisor Dr Per Pettersson for sharing his broad knowledge in uncertainty quantification. Unfortunately, due to the limited time frame, we never found a suitable project collaboration during these years. I also want to thank Prof. Gianluca Iaccarino and the researchers at the Center for turbulence research at Stanford for giving me the opportunity to attend their biannual summer program 2016.

I want to thank all $\mathrm{PhD}$ students at MAI who have influenced this experience in one way or the other. Especially, I want to mention Samira, Andrea, Viktor, Oskar, Tomas, Cristina, Fredrik, Fatemeh, Hannes and Jolanta for all the nice moments and for making life both in- and outside of work enjoyable. In particular, I want to thank Andrea for our many discussions about everything from research to Italian football and for your ability to light up any workday with your great sense of humour. Last, but not least, I should thank Samira, whom I've shared office with for more than three years. Thank you for having been a better roommate I've ever could have wished for.

Finally, I thank my family, for all the moral support and encouragements. Thank you for always being there for me if I ever needed it. I cannot express my gratitude enough, but you should know that I love you more than you'll ever know.

Markus Wahlsten Linköping, 2018 



\section{Contents}

Abstract $\quad$ v

Sammanfattning på svenska vii

List of Papers ix

Acknowledgements $\quad$ xi

1 Introduction 1

2 Well-posedness and stability 3

2.1 Well-posedness . . . . . . . . . . . . . . . . 3

2.2 Stability ........................... 4

3 The computational technique $\quad 7$

3.1 The continuous problem . . . . . . . . . . . . 7

3.1.1 Strong imposition of boundary conditions . . . . . . . 7

3.1.2 Weak imposition of boundary conditions . . . . . . . . 7

3.2 The discrete problem . . . . . . . . . . . . . . 8

3.2 .1 SBP operators ................ 8

3.2.2 The semi-discrete problem . . . . . . . . . . . 9

3.3 Boundary conditions . . . . . . . . . . . . . 10

3.3.1 A model problem . . . . . . . . . . . . . . 10

3.4 Transforming the computational domain . . . . . . . . . . . 12

3.4.1 A model problem . . . . . . . . . . . . 12

4 Uncertainty Quantification 13

4.1 Intrusive methods . . . . . . . . . . . . . . . . . 13

4.1 .1 Polynomial chaos . . . . . . . . . . . . . . 13

4.1.2 Galerkin projection .............. 14

4.2 Non-intrusive methods . . . . . . . . . . . . . . . . . 15

4.2.1 Numerical integration . . . . . . . . . . . 15

5 Variance reduction $\quad 17$

5.1 A model problem . . . . . . . . . . . . . . 17 
6 Coupling different UQ methods $\quad 19$

6.1 Domain decomposition . . . . . . . . . . . . . . . . . . . 19

$\begin{array}{lll}7 & \text { Summary of papers } & 21\end{array}$

References $\quad \mathbf{2 4}$

I. Variance reduction through robust design of boundary conditions for stochastic hyperbolic systems of equations . . . . . . . 27

II. Robust Boundary Conditions for Stochastic Incompletely Parabolic Systems of Equations . . . . . . . . . . . . . 51

III. The effect of uncertain geometries on advection-diffusion of scalar

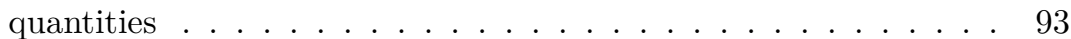

IV. Stochastic Galerkin Projection and Numerical Integration for Stochastic Investigations of the Viscous Burgers' Equation . . . . . . . . 117

V. An efficient hybrid method for uncertainty quantification . . . . . . 133 


\section{1}

\section{Introduction}

Partial differential equations (PDE's) are widely used models describing complex phenomena in physics, chemistry, biology, etc. The parameters and input data in these models are often not perfectly known, and hence to obtain a reliable solution, the uncertainties in these quantities must be addressed. The fairly recently developed methodology of uncertainty quantification (UQ) [25] addresses this issue. The methodology aims to quantify the uncertainty in the output, often in terms of mean value and variance, given uncertainties in the input and parameters of the model.

UQ methods used for solving PDE's with uncertainties can generally be categorized in intrusive and non-intrusive methods. Non-intrusive methods [32, 15] rely on multiple evaluations of an existing deterministic solver augmented with a particular set of random inputs. Quadrature techniques can then be used to obtain statistics of interest. Intrusive methods [10, 29] are generally based on polynomial chaos expansions, leading to systems of equations for the expansion coefficients. Semi-intrusive methods [3, 2] do exist, usually requiring a limited amount of modification of the deterministic code.

The first part of this thesis is dedicated to the construction of variance reducing boundary conditions. A connection between the choice of boundary conditions and the variance of the solution is established. The analysis is conducted on both a hyperbolic and incompletely parabolic system of equations. As applications, problems in fluid mechanics [17] and electromagnetics [18], governed by the Euler, Navier-Stokes and Maxwell's equations are considered.

The second part of this thesis consists of a study of how uncertainties in the geometry affects the solution. These uncertainties can originate from imprecise manufacturing machines, non-perfect mesh generators or an inaccurate computer-aided design (CAD) software. An application in heat transfer [23, 26] is considered, and the statistical quantities mean and variance are computed in a non-intrusive way for varying frequency and amplitude of the uncertainty in the geometry.

The final part of this thesis is devoted to the comparison and coupling between UQ methods. The intrusive polynomial chaos expansion with stochastic Galerkin projection technique $[24,16]$ is compared with the non-intrusive numerical integration technique $[12,27]$ in terms of efficiency for the viscous Burgers' 
equation. Furthermore, a stable procedure for coupling different UQ methods in space is constructed. Consequently, different UQ methods can be used in different regions of the computational domain. This allows for potential efficiency gains by utilizing the UQ methods where they are most efficient.

The introductory part of this thesis is organized as: Chapter 2 introduces the fundamental concepts stability and well-posedness. In Chapter 3, the main analytical tools when studying PDE's i.e. the energy method, the derivation of well-posed boundary conditions and geometry transformations are presented. Moreover, the summation-by-parts operators and simultaneous approximation terms (SBP-SAT) framework [30, 13, 28] on which all numerical approximations in this thesis are based on, are introduced. The fundamentals of the UQ methods used in Paper IV and Paper $V$ are presented in Chapter 4. Chapter 5 presents a brief introduction to the concept of variance reduction which is the main topic in Paper I and Paper II. Finally, Chapter 6 introduces the idea of coupling the different UQ methods described in Chapter 4, through domain decomposition. 


\section{Well-posedness and stability}

Two essential concepts in the theory of partial differential equations (PDE's) and their numerical approximations are well-posedness and stability. In this chapter we provide a brief introduction of these concepts.

\subsection{Well-posedness}

Consider a general linear initial boundary value problem posed on the domain $\Omega$

$$
\begin{array}{rlll}
u_{t}+\mathcal{P}(u) & =F, & x \in \Omega, & t>0 \\
\mathcal{L}(u) & =g, & x \in \partial \Omega, & t>0 \\
u & =f, & x \in \Omega, & t=0
\end{array}
$$

where $u$ denotes the solution, $\mathcal{P}$ is a spatial differential operator and $\mathcal{L}$ a boundary operator defined on the boundary $\partial \Omega$. The functions $F=F(x, t), f=f(x)$ and $g=g(x, t)$ are given forcing, initial and boundary data, respectively. The problem (2.1) is well-posed (assuming that the data is sufficiently smooth) if a unique solution exists and can be bounded by data. Two formal definitions of well-posedness are given below.

Definition 2.1.1. The problem (2.1) with zero boundary data and forcing function is well-posed if a unique solution exists and satisfies

$$
\|u(\cdot, t)\|_{I}^{2} \leq K(t)\|f\|_{I I}^{2}
$$

where $K$ is bounded for finite times $t$ and independent of the solution and initial data. The norms $\|\cdot\|_{I}$ and $\|\cdot\|_{I I}$ are in general the same.

Definition 2.1.2. The problem (2.1) is strongly well-posed if a unique solution $u$ exists and satisfies

$$
\|u(\cdot, t)\|_{I}^{2} \leq K(t)\left(\|f\|_{I I}^{2}+\|F\|_{I I I}^{2}+\|g\|_{I V}^{2}\right) .
$$

In (2.2), $K$ is bounded (for finite times), and independent of the solution and data. The norms $\|\cdot\|_{I, I I, I I I}$ involved in (2.2) are in general the same, while $\|\cdot\|_{I V}$ may differ. 
To illustrate the significance of well-posedness, we introduce a perturbed version of $(2.1)$

$$
\begin{aligned}
& v_{t}+\mathcal{P}(v)=F+\delta F, \quad x \in \Omega, \quad t>0, \\
& \mathcal{L}(v)=g+\delta g, \quad x \in \partial \Omega, \quad t>0, \\
& v=f+\delta f, \quad x \in \Omega, \quad t=0,
\end{aligned}
$$

where $\delta F, \delta f$ and $\delta g$ are small perturbations of the forcing, initial and boundary data. By subtracting (2.1) from (2.3), and letting $w=v-u$, we obtain

$$
\begin{aligned}
& w_{t}+\mathcal{P}(w)=\delta F, \quad x \in \Omega, \quad t>0, \\
& \mathcal{L}(w)=\delta g, \quad x \in \partial \Omega, \quad t>0, \\
& w=\delta f, \quad x \in \Omega, \quad t=0 .
\end{aligned}
$$

If (2.1) is well-posed, then also (2.4) is, and satisfies the estimate

$$
\|w(\cdot, t)\|_{I}^{2} \leq K(t)\left(\|\delta f\|_{I I}^{2}+\|\delta F\|_{I I I}^{2}+\|\delta g\|_{I V}^{2}\right) .
$$

From (2.5), we conclude that small perturbations in the data, results in small perturbations in the solution. Furthermore, uniqueness follows directly from (2.5), by letting $\delta F=\delta f=\delta g=0$, leading to $w=u-v=0$.

\subsection{Stability}

Let us now consider the semi-discrete (discretized in space but not in time) version of $(2.1)$

$$
\begin{aligned}
\mathbf{u}_{t}+\mathbf{D}_{h}(\mathbf{u}, \mathbf{g}) & =\mathbf{F}, \quad t>0 \\
\mathbf{u} & =\mathbf{f}, \quad t=0
\end{aligned}
$$

where the vector $\mathbf{u}$ denotes the numerical solution. Further, $h$ denotes the grid spacing and $\mathbf{D}_{h}$ denotes a discrete spatial operator approximating the continuous differential operator $\mathcal{P}$ augmented with the boundary operator $\mathcal{L}$. The discrete forcing, initial and boundary data $\mathbf{F}, \mathbf{f}$ and $\mathbf{g}$ are obtained by projecting their continuous counterparts $F, f$ and $g$ onto the spatial grid.

Definition 2.2.1. The problem (2.6) with zero boundary data and forcing function is stable if

$$
\|\mathbf{u}(t)\|_{I_{h}}^{2} \leq \mathbf{K}(t)\|\mathbf{f}\|_{I I_{h}}^{2}
$$

holds. In (2.7), $\mathbf{K}$ is bounded (for finite times) and independent of the solution $\mathbf{u}$, the grid spacing and the initial data.

Definition 2.2.2. The problem (2.6) is strongly stable if

$$
\|\mathbf{u}(t)\|_{I_{h}}^{2} \leq \mathbf{K}(t)\left(\|\mathbf{f}\|_{I I_{h}}^{2}+\|\mathbf{F}\|_{I I I_{h}}^{2}+\|\mathbf{g}\|_{I V_{h}}^{2}\right)
$$

holds. In (2.8), $\mathbf{K}$ is bounded (for finite times) and independent of the numerical solution, the grid spacing and the data. 
We can, as in the continuous setting, subtract a perturbed problem (with solution $\mathbf{v}$ ) from (2.6), and define $\mathbf{w}=\mathbf{v}-\mathbf{u}$. If the problem (2.6) is strongly stable, then $\mathbf{w}$ satisfies the estimate

$$
\|\mathbf{w}(t)\|_{I_{h}}^{2} \leq \mathbf{K}(t)\left(\|\delta \mathbf{f}\|_{I I_{h}}^{2}+\|\delta \mathbf{F}\|_{I I I_{h}}^{2}+\|\delta \mathbf{g}\|_{I V_{h}}^{2}\right) .
$$

The estimate (2.9) shows that small variations in the data results in small variations in the numerical solution, for a strongly stable problem. 



\section{The computational technique}

In this chapter we introduce the analytical and numerical techniques used throughout this thesis.

\subsection{The continuous problem}

Here, we show how the energy method can be used to prove well-posedness. The method is exemplified on a simple model problem.

\subsubsection{Strong imposition of boundary conditions}

Consider the scalar advection problem in one dimension

$$
\begin{aligned}
& u_{t}+a u_{x}=0, \quad x \in(0,1) \quad t>0, \\
& u=g(t), \quad x=0, \quad t>0, \\
& u=f(x), \quad x \in(0,1), \quad t=0,
\end{aligned}
$$

where $a>0$. The initial and boundary data are denoted by $f$ and $g$, respectively. Next, we introduce the inner product with a corresponding norm

$$
\langle u, v\rangle=\int_{0}^{1} u v d x, \quad\|u\|^{2}=\langle u, u\rangle .
$$

Applying the energy method (multiplying by the solution and integrating by parts) to (3.1) and imposing the boundary conditions results in

$$
\frac{d}{d t}\|u\|^{2}=a g(t)^{2}-a u(1, t)^{2} .
$$

From (3.2), we conclude that an energy estimate of the form (2.2) is obtained after integration in time.

\subsubsection{Weak imposition of boundary conditions}

Instead of imposing the boundary conditions strongly, one can impose them weakly as

$$
\begin{aligned}
& u_{t}+a u_{x}=a L(g(t)-u), \quad x \in(0,1), \quad t>0, \\
& u=f(x), \quad x \in(0,1), \quad t=0,
\end{aligned}
$$


where $L$ is a so-called lifting operator [4], satisfying

$$
\int_{0}^{1} \phi L(\psi) d x=\left.\phi \psi\right|_{x=0} .
$$

Applying the energy method to (3.3) gives

$$
\frac{d}{d t}\|u\|^{2}=a g(t)^{2}-a u(1, t)^{2}-a(u(0, t)-g(t))^{2} .
$$

Note the similarity between (3.4) and (3.2).

\subsection{The discrete problem}

The numerical formulations throughout this thesis are based on summationby-parts operators (SBP) and simultaneous approximation terms (SAT). This section introduces the SBP-SAT framework and shows how it is designed to mimic the continuous energy rates in (3.2) and (3.4).

\subsubsection{SBP operators}

We start by partitioning an interval $[a, b]$ into $N+1$ equidistant grid points with spacing $h$ such that $\mathbf{x}=\left[x_{0}, \ldots, x_{N}\right]$, where $x_{k}=a+k h$. The discrete approximation of a continuous function $u=u(x)$ is denoted by $\mathbf{u}=\left[\mathbf{u}_{0}, \ldots, \mathbf{u}_{N}\right]$, where $\mathbf{u}_{k} \approx u\left(x_{k}\right)$.

An SBP operators can be defined in the following way

Definition 3.2.1. A matrix $\mathbf{D}=\mathbf{P}^{-1} \mathbf{Q} \in \mathbb{R}^{(N+1) \times(N+1)}$ approximating the first derivative $\frac{\partial}{\partial x}$ of order $q$ is called an $S B P$ operator if

- $\mathbf{D} \mathbf{x}^{k}=k \mathbf{x}^{k-1}, \quad k=0, \ldots, q$,

- $\mathbf{P}$ is symmetric and positive definite.

- $\mathbf{Q}+\mathbf{Q}^{T}=\mathbf{B}=\mathbf{E}_{N}-\mathbf{E}_{0}=\operatorname{diag}(-1,0, \ldots, 0,1)$,

where $\mathbf{E}_{0}$ and $\mathbf{E}_{N}$ are zero matrices of dimension $N+1$ except that element $(1,1)$ and $(N+1, N+1)$ are equal to one, respectively.

The SBP operators are constructed to mimic the integration by parts property

$$
\left\langle u, v_{x}\right\rangle=\left.u v\right|^{b}-\left.u v\right|_{a}+\left\langle u_{x}, v\right\rangle \quad \text { with }\langle u, v\rangle=\int_{a}^{b} u v d x
$$

in the discrete setting, i.e.

$$
\langle\mathbf{u}, \mathbf{D v}\rangle_{\mathbf{P}}=\mathbf{u}_{N} \mathbf{v}_{N}-\mathbf{u}_{0} \mathbf{v}_{0}+\langle\mathbf{D u}, \mathbf{v}\rangle_{\mathbf{P}} \quad \text { with } \quad\langle\mathbf{u}, \mathbf{v}\rangle_{\mathbf{P}}=\mathbf{u}^{T} \mathbf{P} \mathbf{v}
$$


The discrete inner product $\langle\mathbf{u}, \mathbf{v}\rangle_{\mathbf{P}}$ also induces the norm $\|\mathbf{u}\|_{\mathbf{P}}^{2}=\mathbf{u}^{T} \mathbf{P u}$, which approximates the continuous $L^{2}$-norm $\|u\|^{2}=\int_{a}^{b} u^{2} d x$.

Extending SBP operators to higher dimensions can be done in a straightforward manner using Kronecker products. The Kronecker product $\otimes$ is defined as

$$
A \otimes B=\left[\begin{array}{ccc}
A_{11} B & \cdots & A_{1 n} B \\
\vdots & \ddots & \vdots \\
A_{m 1} B & \cdots & A_{m n} B
\end{array}\right],
$$

for two matrices $A \in \mathbb{R}^{m \times n}$ and $B \in \mathbb{R}^{p \times q}$. To illustrate multi-dimensional SBP operators, we partition the domain $(0,1) \times(0,1)$ using $N_{x}+1$ and $N_{y}+1$ points in the $\mathrm{x}$ - and $\mathrm{y}$-direction. Let the numerical solution $\mathbf{u}$ be arranged as

$$
\mathbf{u}=\left[\begin{array}{c}
\mathbf{u}_{0} \\
\vdots \\
\mathbf{u}_{N_{x}}
\end{array}\right], \quad \mathbf{u}_{i}=\left[\begin{array}{c}
\mathbf{u}_{i 0} \\
\vdots \\
\mathbf{u}_{i N_{y}}
\end{array}\right]
$$

where $\mathbf{u}_{i j} \approx u\left(x_{i}, y_{j}\right)$. The multi-dimensional partial derivatives are then approximated as

$$
\left(\mathbf{D}_{x} \otimes I_{N_{y}}\right) \mathbf{u} \approx u_{x}(x, y), \quad\left(I_{N_{x}} \otimes \mathbf{D}_{y}\right) \mathbf{u} \approx u_{y}(x, y) .
$$

In (3.5), $\mathbf{D}_{x, y}$ are SBP operators in the $x$ - and $y$-direction, respectively. Further, $I_{N_{x, y}}$ denotes identity matrices of dimension $N_{x, y}+1$. The design of partial derivatives in even higher dimensions follows the same pattern.

Approximating higher-order derivatives can be done by multiplying the first derivative multiple times [9], i.e. for the second and third derivatives we get

$$
\mathbf{D}^{2} \mathbf{u} \approx u_{x x}, \quad \mathbf{D}^{3} \mathbf{u} \approx u_{x x x} .
$$

Other, more compact versions of the second derivative operator also exist, see $[14,8]$.

\subsubsection{The semi-discrete problem}

The semi-discrete finite difference approximation of (3.1) based on SBP operators combined with the SAT technique [7] is

$$
\begin{aligned}
\mathbf{u}_{t}+a \mathbf{P}^{-1} \mathbf{Q u} & =a \mathbf{P}^{-1} \mathbf{E}_{0}(\mathbf{g}-\mathbf{u}), \\
\mathbf{u}(0) & =\mathbf{f}
\end{aligned}
$$

The vectors $\mathbf{f}$ and $\mathbf{g}$ contain the initial and boundary data projected onto the spatial grid, respectively. Applying the discrete energy method (multiplying by $\mathbf{u}^{T} \mathbf{P}$, adding the transpose and using the SBP property), results in

$$
\frac{d}{d t}\|\mathbf{u}\|_{\mathbf{P}}^{2}=a \mathbf{g}_{0}^{2}-a \mathbf{u}_{N}^{2}-a\left(\mathbf{u}_{0}-\mathbf{g}_{0}\right)^{2}
$$


From (3.7), we conclude that the solution is bounded by data, and hence strong stability is achieved. Note the similarity between (3.7) and (3.4). The SBP-SAT framework can easily be extended to include time discretizations, see [20, 21].

\subsection{Boundary conditions}

In general, the position, number and form of the boundary conditions are not as trivial to find as the ones in the advection equation (3.1). Deriving well-posed boundary conditions is in fact the most fundamental procedure when solving PDE's. In this chapter we provide a general procedure for deriving well-posed boundary conditions, used throughout this thesis [22, 19]. The technique is exemplified on a hyperbolic system of equations.

\subsubsection{A model problem}

Consider a hyperbolic system of equations in two dimensions

$$
\begin{aligned}
& u_{t}+A u_{x}+B u_{y}=0, \quad(x, y) \in \Omega, \quad t>0, \\
& \mathcal{L}(u)=g(x, y, t), \quad(x, y) \in \partial \Omega, \quad t>0, \\
& u=f(x, y), \quad(x, y) \in \Omega, \quad t=0,
\end{aligned}
$$

where $u=\left[u_{1}(x, y, t), \ldots, u_{M}(x, y, t)\right]$ and $A$ and $B$ are symmetric matrices of dimension $M$. Further, $\mathcal{L}$ denotes the boundary operator defined on the boundary $\partial \Omega$ of the domain $\Omega$. Finally, $f$ and $g$ are the given initial and boundary data to the problem.

Applying the energy method (multiplying with the solution $u$ and integrating in space) to (3.8), results in

$$
\|u\|_{t}^{2}=-\oint_{\partial \Omega} u^{T} \bar{A} u d s
$$

where $\bar{A}=A n_{x}+B n_{y}$ and $n=\left(n_{x}, n_{y}\right)^{T}$ is the outward pointing normal vector to $\partial \Omega$. The matrix $\bar{A}$ is symmetric (since $A$ and $B$ are), and hence can be diagonalized as

$$
\bar{A}=X \Lambda X^{T}, \quad X=\left[X_{+}, X_{-}, X_{0}\right], \quad \Lambda=\operatorname{diag}\left[\Lambda^{+}, \Lambda^{-}, \Lambda^{0}\right] .
$$

Remark 3.3.1. If the system in (3.8) is not symmetric, we assume that it can be symmetrized by multiplying from the left and right with a suitable symmetrizer. Examples of symmetrizers for the Euler and Navier-Stokes equations can be found in [1].

The matrices $\Lambda^{+}, \Lambda^{-}$and $\Lambda^{0}$ consists of the positive, negative and zero eigenvalues respectively, with corresponding eigenvectors $X_{+}, X_{-}$and $X_{0}$.

Next, we make the variable transformation $w=X^{T} u$ in (3.9), which results in

$$
\|u\|_{t}^{2}=-\oint_{\partial \Omega} w^{T} \Lambda w d s .
$$


Remark 3.3.2. The general structure of the boundary terms in (3.11) can be obtained also for more complex problems. The characteristic variables $w$ can potentially contain non-linearities or derivatives of the solution $u$.

By letting $w^{ \pm}=X_{ \pm}^{T} u$ and ignoring the contribution from the zero eigenvalues, we get

$$
\|u\|_{t}^{2}=-\oint_{\partial \Omega}\left[\begin{array}{l}
w^{+} \\
w^{-}
\end{array}\right]^{T}\left[\begin{array}{cc}
\Lambda^{+} & 0 \\
0 & \Lambda^{-}
\end{array}\right]\left[\begin{array}{l}
w^{+} \\
w^{-}
\end{array}\right] d s .
$$

From (3.12), it is clear that the term $\left(w^{-}\right)^{T} \Lambda^{-}\left(w^{-}\right)$might create an energy growth, and hence must be bounded by imposing boundary conditions.

We introduce the following combination of the characteristic variables $w^{+}$and $w^{-}$as boundary conditions

$$
\mathcal{L}(w)=w^{-}-R w^{+}=g,
$$

where our aim is to derive conditions on the matrix $R$ leading to well-posedness. By inserting (3.13) in (3.12), we get

$$
\|u\|_{t}^{2}=-\oint_{\partial \Omega}\left[\begin{array}{c}
w^{+} \\
g
\end{array}\right]^{T}\left[\begin{array}{cc}
R^{T} \Lambda^{-} R+\Lambda^{+} & R^{T} \Lambda^{-} \\
\left(R^{T} \Lambda^{-}\right)^{T} & \Lambda^{-}
\end{array}\right]\left[\begin{array}{c}
w^{+} \\
g
\end{array}\right] d s .
$$

We add and subtract a (yet unknown) bounded matrix $G$ in (3.14) to obtain

$$
\|u\|_{t}^{2}=-\oint_{\partial \Omega}\left[\begin{array}{c}
w^{+} \\
g
\end{array}\right]^{T} M\left[\begin{array}{c}
w^{+} \\
g
\end{array}\right] d s+\oint_{\partial \Omega} g^{T}\left(\left|\Lambda^{-}\right|+G\right) g d s,
$$

where

$$
M=\left[\begin{array}{cc}
R^{T} \Lambda^{-} R+\Lambda^{+} & R^{T} \Lambda^{-} \\
\left(R^{T} \Lambda^{-}\right)^{T} & G
\end{array}\right] .
$$

In order for $M$ to be positive semi-definite, we need

$$
R^{T} \Lambda^{-} R+\Lambda^{+}>0,
$$

and that the Schur complement $G-\left(R^{T} \Lambda^{-}\right)^{T}\left(R^{T} \Lambda^{-} R+\Lambda^{+}\right)^{-1} R^{T} \Lambda^{-}$is positive semi-definite. The choice

$$
G=\left(R^{T} \Lambda^{-}\right)^{T}\left(R^{T} \Lambda^{-} R+\Lambda^{+}\right)^{-1} R^{T} \Lambda^{-},
$$

makes the matrix $M$ positive semi-definite. Finally, integrating (3.15) in time results in

$$
\|u(T)\|^{2} \leq\|f\|^{2}+\int_{0}^{T} \oint_{\partial \Omega} g^{T}\left(\left|\Lambda^{-}\right|+G\right) g d s d t .
$$

To summarize: we can obtain the energy estimate (3.19), if $R$ satisfies (3.17). Note that the choice of $G$ as in (3.18) can always be made if (3.17) holds.

Remark 3.3.3. With homogeneous data $(g=0)$, the condition on $R$ can be relaxed to

$$
R^{T} \Lambda^{-} R+\Lambda^{+} \geq 0
$$




\subsection{Transforming the computational domain}

In this chapter we give a brief introduction to the transformation procedure used in Paper III. The transformation procedure is needed due to the fact that the SBP operators are constructed for Cartesian grids.

\subsubsection{A model problem}

Consider the constant coefficient two-dimensional advection equation

$$
\begin{aligned}
u_{t}+a u_{x}+b u_{y} & =0, & (x, y) \in \Omega, & t>0 \\
\mathcal{L}(u) & =g, & (x, y) \in \partial \Omega, & t>0, \\
u & =f, & (x, y) \in \Omega, & t=0
\end{aligned}
$$

on the curvilinear domain $\Omega$, with boundary $\partial \Omega$. We transform the domain $\Omega$ to the unit square using a curvilinear transformation

$$
\begin{aligned}
& x=x(\xi, \eta), \quad \xi=\xi(x, y) \\
& y=y(\xi, \eta), \quad \eta=\eta(x, y)
\end{aligned}
$$

where $(\xi, \eta) \in[0,1] \times[0,1]$. The Jacobian matrix of the transformation is

$$
[J]=\left[\begin{array}{ll}
x_{\xi} & y_{\xi} \\
x_{\eta} & y_{\eta}
\end{array}\right]
$$

Applying the chain rule to (3.21) and multiplying by $J=\operatorname{det}([J])=x_{\xi} y_{\eta}-x_{\eta} y_{\xi}$, results in

$$
J u_{t}+J\left(a \xi_{x}+b \xi_{y}\right) u_{\xi}+J\left(a \eta_{x}+b \eta_{y}\right) u_{\eta}=0 .
$$

Using the product rule and the geometric conservation law $[6]\left(\left(J \xi_{x}\right)_{\xi}+\left(J \eta_{x}\right)_{\eta}=\right.$ $\left.\left(J \xi_{y}\right)_{\xi}+\left(J \eta_{y}\right)_{\eta}=0\right)$ in $(3.22)$, results in

$$
J u_{t}+\left[J\left(a \xi_{x}+b \xi_{y}\right) u\right]_{\xi}+\left[J\left(a \eta_{x}+b \eta_{y}\right) u\right]_{\eta}=0 .
$$

The resulting transformed problem is

$$
\begin{aligned}
& J u_{t}+(\tilde{a} u)_{\xi}+(\tilde{b} u)_{\eta}=0, \quad(\xi, \eta) \in \Phi, \quad t>0, \\
& \tilde{\mathcal{L}}(u)=g, \quad(\xi, \eta) \in \partial \Phi, \quad t>0, \\
& u=f, \quad(\xi, \eta) \in \Phi, \quad t=0,
\end{aligned}
$$

where $\tilde{a}=J\left(a \xi_{x}+b \xi_{y}\right), \tilde{b}=J\left(a \eta_{x}+b \eta_{y}\right)$ and $\Phi=(0,1) \times(0,1)$. Note that we obtain a system of the same structure as the original problem (3.21) but with different and varying wave speeds $(\tilde{a}$ and $\tilde{b})$, posed on a Cartesian domain. 


\section{Uncertainty Quantification}

Methods for solving PDE's with uncertainties can essentially be divided into intrusive $[24,16]$ and non-intrusive methods $[12,29]$. There are also examples of semi-intrusive methods $[3,2]$, however, they are rare. This chapter gives a brief description of the UQ methods used throughout this thesis.

\subsection{Intrusive methods}

Intrusive methods results in a reformulation of the original problem arising from expanding the solution in an orthogonal basis. Since the intrusive formulation in general is different from the original deterministic formulation, a new code must often be developed.

\subsubsection{Polynomial chaos}

Consider the probability space $\left(\Omega_{\xi}, \mathcal{F}, \mathcal{P}\right)$, where $\Omega_{\xi}, \mathcal{F}$ and $\mathcal{P}$ denotes the sample space, $\sigma$-algebra and probability measure, respectively. We begin by introducing the inner product between two functions $u(\xi)$ and $v(\xi)$, (where $\xi$ denotes a random variable)

$$
\langle u, v\rangle=\int_{\Omega_{\xi}} u v d \mathcal{P}(\xi),
$$

which induces the norm

$$
\|u\|_{L^{2}\left(\Omega_{\xi}, \mathcal{P}\right)}^{2}=\langle u, u\rangle .
$$

A second order random field $u$, i.e. satisfying $\int_{\Omega_{\xi}} u^{2} d \mathcal{P}(\xi)<\infty$ [33], can be expanded as

$$
u(\xi)=\sum_{k=0}^{\infty} u_{k} \psi_{k}(\xi),
$$

where $\left\{\psi_{k}(\xi)\right\}_{k=0}^{\infty}$ is a set of orthonormal basis functions in $L^{2}\left(\Omega_{\xi}\right)$ with respect to the inner product (4.1). The coefficients $\left\{u_{k}\right\}_{k=0}^{\infty}$ are given by the projection

$$
u_{k}=\left\langle\psi_{k}, u\right\rangle \text {. }
$$


The statistical quantities, such as the mean and variance are given by

$$
\mathbb{E}[u]=\int_{\Omega_{\xi}} \sum_{k=0}^{\infty} u_{k} \psi_{k} d \mathcal{P}(\xi)=\sum_{k=0}^{\infty} u_{k} \int_{\Omega_{\xi}} \psi_{k} d \mathcal{P}(\xi)=u_{0}
$$

and

$$
\begin{aligned}
\operatorname{Var}[u] & =\mathbb{E}\left[u^{2}\right]-\mathbb{E}[u]^{2}=\int_{\Omega_{\xi}}\left(\sum_{k=0}^{\infty} u_{k} \psi_{k}\right)^{2} d \mathcal{P}(\xi)-\left(\int_{\Omega_{\xi}} \sum_{k=0}^{\infty} u_{k} \psi_{k} d \mathcal{P}(\xi)\right)^{2} \\
& =\sum_{k=0}^{\infty} u_{k}^{2}-u_{0}^{2}=\sum_{k=1}^{\infty} u_{k}^{2}
\end{aligned}
$$

respectively. In (4.4), the orthonormality property of the basis functions have been used.

\subsubsection{Galerkin projection}

To illustrate the Galerkin projection procedure, consider the scalar advection equation with random data

$$
\begin{aligned}
& u_{t}+a u_{x}=F(x, t, \xi), \quad x \in(0,1), \quad t>0, \\
& u=g(t, \xi), \quad x=0, \quad t>0, \\
& u=f(x, \xi), \quad x \in(0,1), \quad t=0,
\end{aligned}
$$

where $u=u(x, t, \xi)$ and $a=a(x, t)>0$. We start by expanding the solution $u$ in a truncated series

$$
u(x, t, \xi)=\sum_{k=0}^{M} u_{k}(x, t) \psi_{k}(\xi)
$$

Inserting (4.6) in (4.5) results in

$$
\begin{aligned}
& \sum_{k=0}^{M}\left(u_{k}\right)_{t} \psi_{k}+a \sum_{k=0}^{M}\left(u_{k}\right)_{x} \psi_{k}=F(x, t, \xi), \quad x \in(0,1), \quad t>0 \\
& \sum_{k=0}^{M} u_{k} \psi_{k}=g(t, \xi), \quad x=0, \quad t>0 \\
& \sum_{k=0}^{M} u_{k} \psi_{k}=f(x, \xi), \quad x \in(0,1), \quad t=0 .
\end{aligned}
$$


Multiplying (4.7) by $\psi_{l}$ and integrating over $\Omega_{\xi}$ gives

$$
\begin{aligned}
& \sum_{k=0}^{M}\left(u_{k}\right)_{t}\left\langle\psi_{k}, \psi_{l}\right\rangle+a \sum_{k=0}^{M}\left(u_{k}\right)_{x}\left\langle\psi_{k}, \psi_{l}\right\rangle=\left\langle F(x, t, \xi), \psi_{l}\right\rangle, \quad x \in(0,1), \quad t>0, \\
& \sum_{k=0}^{M} u_{k}\left\langle\psi_{k}, \psi_{l}\right\rangle=\left\langle g(t, \xi), \psi_{l}\right\rangle, \quad x=0, \quad t>0, \\
& \sum_{k=0}^{M} u_{k}\left\langle\psi_{k}, \psi_{l}\right\rangle=\left\langle f(x, \xi), \psi_{l}\right\rangle, \quad x \in(0,1), \quad t=0,
\end{aligned}
$$

for $l=0,1, \ldots, M$. By using the fact that the basis functions $\left\{\psi_{k}\right\}_{k=0}^{M}$ are orthonormal in $L^{2}\left(\Omega_{\xi}\right)$, i.e. $\left\langle\psi_{i}, \psi_{j}\right\rangle=\delta_{i j}$, in (4.8), we obtain

$$
\begin{aligned}
& \left(u_{l}\right)_{t}+a\left(u_{l}\right)_{x}=\left\langle F(x, t, \xi), \psi_{l}\right\rangle, \quad x \in(0,1), \quad t>0, \\
& u_{l}=\left\langle g(t, \xi), \psi_{l}\right\rangle, \quad x=0, \quad t>0, \\
& u_{l}=\left\langle f(x, \xi), \psi_{l}\right\rangle, \quad x \in(0,1), \quad t=0,
\end{aligned}
$$

for $l=0,1, \ldots, M$. Note that (4.9) consists of an uncoupled system of $M+1$ equations. A non-linear equation or a coefficient $a=a(\xi)$ would result in a coupled system.

\subsection{Non-intrusive methods}

Unlike intrusive methods, non-intrusive methods rely on solving the original problem (in this case (4.5)) multiple times for a fixed sample of stochastic inputs. Statistical quantities such as the mean, variance, skewness, etc. are then computed using these samples. An obvious advantage of non-intrusive methods is that it can be done using the existing deterministic solver, hence no code development is necessary.

\subsubsection{Numerical integration}

Non-intrusive UQ methods rely on approximations of integrals when computing statistics of a given quantity of interest. To approximate these integrals, a numerical integration technique based on quadrature relations can be used. A one-dimensional quadrature relation approximating the integral of $f$ can be written

$$
\int_{a}^{b} f(x) \rho(x) d x \approx \sum_{m=1}^{M} f\left(x^{m}\right) w^{m} .
$$

In (4.10), $\rho(x)>0$ denotes the density function. The integer $M$ denotes the number of quadrature points $x^{m}$ with corresponding positive weights $w^{m}$. The distribution of the quadrature points and choice of weights determines the accuracy of the integral approximation. 
Quadrature relations in higher dimensions can easily be generalized from (4.10), as

$$
\int_{\Omega} f(\vec{x}) \rho(\vec{x}) d \vec{x} \approx \sum_{m_{1}=1}^{M_{1}} \cdots \sum_{m_{p}}^{M_{p}} f\left(x_{1}^{m_{1}}, \ldots, x_{p}^{m_{p}}\right) w^{m_{1}} \cdots w^{m_{p}},
$$

where $\vec{x}=\left[x_{1}, \ldots, x_{p}\right]$ and $\Omega$ is a $p$-dimensional domain, while $x_{i}^{m}$ and $w_{i}^{m}$ are the quadrature point and weights of dimension $i$. The number of quadrature points in dimension $i$ is denoted $M_{i}$. Computing quadratures in higher dimensions is computationally demanding (curse of dimensionality [31]), hence adaptive sparse grids have been developed to reduce these computational efforts $[5]$. 


\section{Variance reduction}

This chapter introduces the variance reduction technique developed in Paper I and Paper II. We show that different boundary conditions with the same data leads to different amounts of uncertainties in the solution.

\subsection{A model problem}

Consider a hyperbolic problem with uncertainties in the initial data

$$
\begin{aligned}
& u_{t}+A u_{x}=0, \quad x \in(0,1), \quad t>0, \\
& \mathcal{L}_{0}(u)=g_{0}(t), \quad x=0, \quad t>0, \\
& \mathcal{L}_{1}(u)=g_{1}(t), \quad x=1 \quad t>0, \\
& u=f(x, \xi), \quad x \in(0,1), \quad t=0,
\end{aligned}
$$

where for simplicity $[A]_{11}=[A]_{22}=0$ and $[A]_{12}=[A]_{21}=1$. The initial data $f$ can be split into the mean $(\mathbb{E}[f])$ and variation from the mean $(\delta f)$. The expected value (or mean) of $f$ is defined as $\mathbb{E}[f]=\int_{\Omega_{\xi}} f d \rho(\xi)$, where $\rho$ is the probability density function. By taking the expected value of (5.1), we get

$$
\begin{aligned}
& v_{t}+A v_{x}=0, \quad x \in(0,1), \quad t>0, \\
& \mathcal{L}_{0}(v)=g_{0}(t), \quad x=0, \quad t>0, \\
& \mathcal{L}_{1}(v)=g_{1}(t), \quad x=1, \quad t>0, \\
& v=\mathbb{E}[f], \quad x \in(0,1), \quad t=0,
\end{aligned}
$$

with $v=\mathbb{E}[u]$. Subtracting (5.2) from (5.1) and letting $e=u-v$ yields

$$
\begin{aligned}
& e_{t}+A e_{x}=0, \quad x \in(0,1), \quad t>0, \\
& \mathcal{L}_{0}(e)=0, \quad x=0, \quad t>0, \\
& \mathcal{L}_{1}(e)=0, \quad x=1, \quad t>0, \\
& e=\delta f, \quad x \in(0,1), \quad t=0 .
\end{aligned}
$$

By following the path in Section 3.3, we apply the energy method to (5.3) and obtain

$$
\|e\|_{t}^{2}=-\left.e^{T} A e\right|_{0} ^{1}=-\left.w^{T} \Lambda w\right|_{0} ^{1}=-\left(w^{+}\right)^{2}+\left.\left(w^{-}\right)^{2}\right|^{1}+\left(w^{+}\right)^{2}-\left.\left(w^{-}\right)^{2}\right|_{0},
$$

where $A=X \Lambda X^{T}, w=X^{T} e$ and

$$
\begin{aligned}
e & =\left[e_{1}, e_{2}\right]^{T}, \quad w=\left[w^{+}, w^{-}\right]^{T}, \quad w^{+}=\frac{e_{1}+e_{2}}{\sqrt{2}}, \\
w^{-} & =\frac{-e_{1}+e_{2}}{\sqrt{2}}, \quad \Lambda=\left[\begin{array}{cc}
1 & 0 \\
0 & -1
\end{array}\right], \quad\|e\|^{2}=\int_{0}^{1} e^{T} e d x .
\end{aligned}
$$


The boundary conditions imposed are of the general form (3.13), that is

$$
\mathcal{L}_{0}(w)=w^{+}-R_{0} w^{-}=0, \quad \mathcal{L}_{1}(w)=w^{-}-R_{1} w^{+}=0 .
$$

Inserting the boundary conditions (5.5) in (5.4) results in

$$
\|e\|_{t}^{2}=\left.\left(R_{1}^{2}-1\right)\left(w^{+}\right)^{2}\right|^{1}+\left.\left(R_{0}^{2}-1\right)\left(w^{-}\right)^{2}\right|_{0} .
$$

Note the similarity between (5.6) and (3.14) with homogeneous data $(g=0)$.

Furthermore, we note

$$
\begin{aligned}
\mathbb{E}\left[\|e\|^{2}\right] & =\mathbb{E}\left[\int_{0}^{1} e^{2} d x\right]=\int_{0}^{1} \mathbb{E}\left[e^{2}\right] d x=\int_{0}^{1} \mathbb{E}\left[(u-\mathbb{E}[u])^{2}\right] d x \\
& =\int_{0}^{1} \operatorname{Var}[u] d x=\|\operatorname{Var}[u]\|_{1}
\end{aligned}
$$

where we have used the fact that $\operatorname{Var}[u]=\mathbb{E}\left[u^{2}\right]-\mathbb{E}[u]^{2}$ and that $\|\cdot\|_{1}$ denotes the $L^{1}$-norm. Taking the expected value of (5.6) and using (5.7), results in

$$
\frac{d}{d t}\|\operatorname{Var}[u]\|_{1}=\left.\left(R_{1}^{2}-1\right) \operatorname{Var}\left[w^{+}\right]\right|^{1}+\left.\left(R_{0}^{2}-1\right) \operatorname{Var}\left[w^{-}\right]\right|_{0} .
$$

From (5.8) we have a clear description of how the variance of the solution relates to the choice of boundary conditions. It varies depending on the choices of $R_{0}$ and $R_{1}$.

Remark 5.1.1. If in (5.7), e and $u$ are vectors with $M$ components then $\|\operatorname{Var}[u]\|_{1}=\int_{0}^{1} \sum_{k=1}^{M} \operatorname{Var}\left[u_{i}\right] d x$. 


\section{Coupling different UQ methods}

The general conclusion from Paper IV was that the UQ methods mentioned in Chapter 4 are efficient for different types of problems. It was concluded that polynomial chaos with stochastic Galerkin projection seems more efficient than numerical integration for slowly varying problems, and vice versa for fastly varying problems. Given this indication, we construct a hybrid method by combining the strengths of the UQ methods to potentially increase the efficiency. This chapter introduces the idea behind Paper $V$ where a hybrid method is constructed by splitting the spatial domain into different parts and coupling the UQ methods at the interface between these domains in a stable way.

\subsection{Domain decomposition}

Consider again problem (4.1) posed on the domain $\Omega=(-1,1)$. The splitting of the domain $\Omega$ at $\Gamma=\{0\}$, results in

$$
\begin{aligned}
& v_{t}+a v_{x}=0, \quad x \in \Omega_{R}, \quad t>0, \\
& v=g_{R}(t, \xi), \quad x \in \partial \Omega_{R} \backslash \Gamma, \quad t>0, \\
& v=f_{R}(x, \xi), \quad x \in \Omega_{R}, \quad t=0, \\
& v=w, \quad x \in \Gamma, \quad t>0, \\
& w_{t}+a w_{x}=0, \quad x \in \Omega_{L}, \quad t>0, \\
& w=g_{L}(t, \xi), \quad x \in \partial \Omega_{L} \backslash \Gamma, \quad t>0, \\
& w=f_{L}(x, \xi), \quad x \in \Omega_{L}, \quad t=0,
\end{aligned}
$$

where $v$ and $w$ are the solutions at the domains $\Omega_{R}=(0,1)$ and $\Omega_{L}=(-1,0)$, respectively, see Figure 6.1.

The idea outlined in Paper $V$ is to couple the UQ methods numerical integration and polynomial chaos with stochastic Galerkin projection in a numerically stable manner at the interface $\Gamma$. This means that information must be exchanged between the solutions $v$ and $w$ at the interface. Moreover, a special kind of projection operators, projecting the solutions through the interface is required. The coupling also allows that the same UQ method is used in both domains but with different numbers of stochastic grid points (or coefficients when using stochastic Galerkin). 


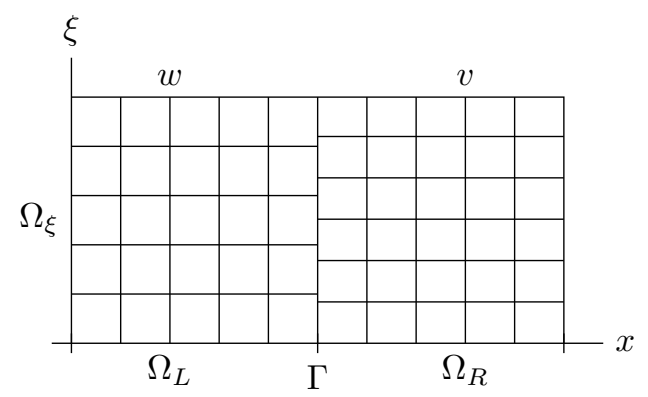

Figure 6.1: An illustration of the computational domains, where the solutions $v$ and $w$ are defined in $\Omega_{R}$ and $\Omega_{L}$, respectively. 


\section{Summary of papers}

\section{PAPER I: Variance reduction through robust de- sign of boundary conditions for stochastic hyper- bolic systems of equations}

In Paper $I$, we consider a general hyperbolic system with random data. We start by deriving a family of strongly well-posed boundary conditions. The problem was discretized using a provably strongly stable numerical formulation based on SBP operators with a weak imposition of the boundary conditions. Using the energy method, a complete description of how the choice of boundary conditions affects the variance of the solution was derived.

Several types of boundary conditions were compared in different scenarios in terms of the norm of the variance. The scenarios considered were i) perfect boundary knowledge ii) decaying variance on the boundary iii) large nondecaying variance on the boundary. Numerical experiments were performed on a model problem and applications in fluid mechanics and electromagnetics. The statistical quantities such as the mean and variance were calculated using quadrature rules. It was shown that the characteristic boundary conditions in general were a good choice in terms of variance reduction.

\section{PAPER II: Robust Boundary Conditions for Stoc- hastic Incompletely Parabolic Systems of Equa- tions}

In Paper II, we extend the analysis in Paper $I$ to general incompletely parabolic systems with random data. A formulation relating the choice of strongly wellposed boundary conditions to the evolution of the variance of the solution was derived. It was further proven that the expected value of the solution for linear problems is independent of the choice of boundary condition. An incompletely parabolic problem was analyzed analytically as well as numerically through numerical experiments. It was shown that the dissipation has a reducing effect on the size of variance of the solution.

The different scenarios studied included i) large, ii) decaying and iii) zero variance in the boundary data. As an application, the one-dimensional Navier- 
Stokes equations were considered. Five different boundary conditions were compared numerically. It was concluded that the generalized characteristic boundary conditions are optimal in terms of variance reduction for zero or decaying variance in the boundary data. For large variances in the boundary data however, no general conclusions could be drawn.

\section{PAPER III: The effect of uncertain geometries on advection-diffusion of scalar quantities}

In Paper III, a two-dimensional advection-diffusion equation posed on an uncertain geometry was considered. The effects on the solution by imposing the boundary data on the uncertain boundary was investigated. The problem posed on an uncertain geometry was transformed to a stochastically varying coefficient problem posed on the unit square. Statistical quantities such as the mean and variance of the solution were computed using numerical integration.

As an application, heat transfer on a randomly varying boundary was considered. Mean and variances were computed when varying the amplitude and frequency of the uncertain geometry. It was concluded that an increased amplitude leads to an increased variance and that a higher frequency leads to a larger variance. The correlation length of the geometry was also studied but numerical experiments indicated no significant change in the variance.

\section{PAPER IV: Stochastic Galerkin Projection and Numerical Integration for Stochastic Investigations of the Viscous Burgers' Equation}

Paper IV deals with the comparison between intrusive and non-intrusive UQ methods. The UQ methods considered were polynomial chaos with stochastic Galerkin projection and numerical integration. The comparison was performed on the non-linear viscous Burgers' equation where non-linear boundary conditions leading to an energy estimate were derived.

The continuous problem was discretized using a provably stable high-order finite difference formulation based on the SBP-SAT framework on which the UQ methods were employed. As a measure of comparison, the error of the variance of the solution was computed. Numerical experiments indicated that numerical integration was advantageous for fastly varying problems, while polynomial chaos performed better for slowly varying problems.

\section{PAPER V: An efficient hybrid method for uncer- tainty quantification}

In Paper $V$, a provably stable hybrid method for UQ was constructed. The coupling between the non-intrusive numerical integration and intrusive polynomial chaos with stochastic Galerkin extends the work in [11]. The coupling procedure 
was developed for a general hyperbolic system. The numerical approximation was based on a high-order finite difference formulation using SBP operators with a weak imposition of the boundary conditions. Various couplings were demonstrated on a hyperbolic model problem, corroborating the theoretical analysis. 


\section{References}

[1] S. Abarbanel and D. Gottlieb. Optimal time splitting for two- and threedimensional Navier-Stokes equations with mixed derivatives. J COMPUT PHYS, 41:1-43, 1981.

[2] Remi Abgrall and Pietro Marco Congedo. A semi-intrusive deterministic approach to uncertainty quantification in non-linear fluid flow problems. $J$ COMPUT PHYS, 235:828-845, 2013.

[3] Remi Abgrall, Pietro Marco Congedo, Christophe Corre, and Stéphane Galera. A simple semi-intrusive method for uncertainty quantification of shocked flows, comparison with a non-intrusive polynomial chaos method. In ECCOMAS CFD, pages 1-11, 2010.

[4] Douglas N Arnold, Franco Brezzi, Bernardo Cockburn, and L Donatella Marini. Unified analysis of discontinuous Galerkin methods for elliptic problems. SIAM journal on numerical analysis, 39(5):1749-1779, 2002.

[5] H-J Bungartz and Stefan Dirnstorfer. Multivariate quadrature on adaptive sparse grids. Computing, 71(1):89-114, 2003.

[6] Weiming Cao, Weizhang Huang, and Robert D Russell. A moving mesh method based on the geometric conservation law. SIAM Journal on Scientific Computing, 24(1):118-142, 2002.

[7] Mark H Carpenter, David Gottlieb, and Saul Abarbanel. Time-stable boundary conditions for finite-difference schemes solving hyperbolic systems: methodology and application to high-order compact schemes. $J$ COMPUT PHYS, 111(2):220-236, 1994.

[8] M.H. Carpenter, J. Nordström, and D. Gottlieb. A Stable and Conservative Interface Treatment of Arbitrary Spatial Accuracy. J COMPUT PHYS, 148:341-365, 1999.

[9] Hannes Frenander and Jan Nordström. Spurious solutions for the advection-diffusion equation using wide stencils for approximating the second derivative. Numerical Methods for Partial Differential Equations, $34(2): 501-517,2018$.

[10] David Gottlieb and Dongbin Xiu. Galerkin method for wave equations with uncertain coefficients. Commun. Comput. Phys, 3(2):505-518, 2008.

[11] Jeremy E Kozdon and Lucas C Wilcox. Stable coupling of nonconforming, high-order finite difference methods. SIAM Journal on Scientific Computing, 38(2):A923-A952, 2016.

[12] OP Le Maître and Omar M Knio. Non-intrusive methods. In Spectral Methods for Uncertainty Quantification, pages 45-72. Springer, 2010. 
[13] K. Mattsson. Summation-by-Parts Operators for High Order Finite Difference Methods. Phd thesis, Uppsala University, Depart. of Scientific Computing, Uppsala Univ., Uppsala, Sweden, 2003.

[14] K. Mattsson and J. Nordström. Summation by parts operators for finite difference approximations of second derivatives. J COMPUT PHYS, 199:503$540,2004$.

[15] Siddhartha Mishra, Ch Schwab, and Jonas Šukys. Multi-level Monte Carlo finite volume methods for nonlinear systems of conservation laws in multidimensions. Journal of Computational Physics, 231(8):3365-3388, 2012.

[16] Habib N Najm. Uncertainty quantification and polynomial chaos techniques in computational fluid dynamics. Annual review of fluid mechanics, 41:3552,2009 .

[17] J. Nordström and M. H. Carpenter. Boundary and interface conditions for high order finite difference methods applied to the Euler and Navier-Stokes equations. J COMPUT PHYS, 148:621-645, 1999.

[18] J. Nordström, K. Forsberg, C. Adamsson, and P. Eliasson. Finite volume methods, unstructured meshes and strict stability. Applied Numerical Mathematics, 45:453-473, 2003.

[19] Jan Nordström. A roadmap to well posed and stable problems in computational physics. Journal of Scientific Computing, 71(1):365-385, 2017.

[20] Jan Nordström and Tomas Lundquist. Summation-by-parts in time. Journal of Computational Physics, 251:487-499, 2013.

[21] Jan Nordström and Tomas Lundquist. Summation-by-parts in time: the second derivative. SIAM Journal on Scientific Computing, 38(3):A1561A1586, 2016.

[22] Jan Nordström and Magnus Svärd. Well-posed boundary conditions for the Navier-Stokes Equations. SIAM J NUMER ANAL, 43(3):1231-1255, 2005 .

[23] Suhas V Patankar and D Brian Spalding. A calculation procedure for heat, mass and momentum transfer in three-dimensional parabolic flows. In Numerical Prediction of Flow, Heat Transfer, Turbulence and Combustion, pages 54-73. Elsevier, 1983.

[24] M Per Pettersson, Gianluca Iaccarino, and J Nordström. Polynomial Chaos Methods for Hyperbolic Partial Differential Equations. Springer Math Eng. doi, 10:978-3, 2015.

[25] Christopher J Roy and William L Oberkampf. A comprehensive framework for verification, validation, and uncertainty quantification in scientific computing. Computer methods in applied mechanics and engineering, 200(25-28):2131-2144, 2011. 
[26] RA Seban. Skin-friction and heat-transfer characteristics of a laminar boundary layer on a cylinder in axial incompressible flow. Journal of the Aeronautical Sciences, 18(10):671-675, 1951.

[27] Ralph C Smith. Uncertainty quantification: theory, implementation, and applications, volume 12. Siam, 2013.

[28] Bo Strand. Summation by parts for finite difference approximations for d/dx. J COMPUT PHYS, 110(1):47-67, 1994.

[29] TJ Sullivan. Non-intrusive methods. In Introduction to Uncertainty Quantification, pages 277-294. Springer, 2015.

[30] Magnus Svärd and Jan Nordström. Review of summation-by-parts schemes for initial-boundary-value problems. J COMPUT PHYS, 268:17-38, 2014.

[31] Michel Verleysen and Damien François. The curse of dimensionality in data mining and time series prediction. In International Work-Conference on Artificial Neural Networks, pages 758-770. Springer, 2005.

[32] Dongbin Xiu and Jan S Hesthaven. High-order collocation methods for differential equations with random inputs. SIAM Journal on Scientific Computing, 27(3):1118-1139, 2005.

[33] Akiva M Yaglom et al. Second-order homogeneous random fields. In Proceedings of the Fourth Berkeley Symposium on Mathematical Statistics and Probability, Volume 2: Contributions to Probability Theory. The Regents of the University of California, 1961. 


\section{Papers}

The papers associated with this thesis have been removed for copyright reasons. For more details about these see:

http://urn.kb.se/resolve?urn=urn:nbn:se:liu:diva-146966 